\title{
DETECTION OF TEV GAMMA RAYS FROM SN1006
}

\author{
TORU TANIMORI \\ Department of Physics, Tokyo Institute of Technology \\ Ohokayama, Meguro, Tokyo 152, Japan
}

\section{Introduction}

In spite of the recent progress of high energy gamma-ray astronomy, there still remains quite unclear and important problem about the origin of cosmic rays. Supernova remnants (SNRs) are the favoured site for cosmic rays up to $10^{16} \mathrm{eV}$, as they satisfy the requirements such as an energy input rate. But direct supporting evidence is sparse. Recently intense non-thermal Xray emission from the rims of the Type Ia SNR SN1006 (G327.6+14.6) has been observed by ASCA (Koyama et al. 1995)and ROSAT (Willingale et al. 1996), which is considered, by attributing the emission to synchrotron radiation, to be strong evidence of shock acceleration of high energy electrons up to $\sim 100 \mathrm{TeV}$. If so, $\mathrm{TeV}$ gamma rays would also be expected from inverse Compton scattering (IC) of low energy photons (mostly attributable to the $2.7 \mathrm{~K}$ cosmic background photons) by these electrons. By assuming the magnetic field strength $(B)$ in the emission region of the SNR, several theorists ( Pohl 1996; Mastichiadis 1996; Mastichiadis \& de Jager 1996; Yoshida \& Yanagita 1997) calculated the expected spectra of $\mathrm{TeV}$ gamma rays using the observed radio/X-ray spectra. Observation of $\mathrm{TeV}$ gamma rays would thus provide not only the further direct evidence of the existence of very high energy electrons but also the another important information such as the strength of the magnetic field and diffusion coefficient of the shock acceleration. With this motivation, SN1006 was observed by the CANGAROO imaging air Cerenkov telescope in 1996 March and June, also 1997 March and April.

\section{Analysis and Result}

SN1006 was observed with the $3.8 \mathrm{~m}$ diameter Čerenkov imaging telescope of the CANGAROO Collaboration (Patterson \& Kifune 1992; Hara et al. $1993)$ near Woomera, South Australia (136 $47^{\prime} \mathrm{E}$ and31 $\left.01^{\circ} \mathrm{S}\right)$. The 3.8 
$\mathrm{m}$, alt-azimuth mounted, telescope had a $\sim 3 \mathrm{TeV}$ threshold for detecting gamma rays near $70^{\circ}$ elevation in 1996 observation. After 1996 observation the $3.8 \mathrm{~m}$ mirror was recoated in 1996 October, and its reflectivity improved from $45 \%$ to more than $80 \%$, which decreased the threshold energy about a half $(\sim 1.5 \mathrm{TeV})$. SN1006 was observed in 1996 and 1997.

The imaging analysis was carried out using standard parameterization of the elongated shape of the Cerenkov light image (Weekes et al. 1989). The $\alpha$ (image-orientation angle) peak appearing around the origin $\left(\alpha \leq 15^{\circ}\right)$ in the on-source data are attributed to $\gamma$-rays from the target position, and the number of background events under the $\alpha$ peak was estimated from the flat region of the $\alpha$ distribution $\left(30^{\circ}-90^{\circ}\right)$ in the on-source data. This technique has been, to date, applied for the detections of $\mathrm{TeV}$ gamma rays from PSR1706-44 (Kifune et al. 1995) and the nebula surrounding the Crab (Tanimori et al. 1998). From the results on the previously observed objects, the point spread function (PSF) of the CANGAROO telescope is estimated to have a standard deviation of $0^{\circ} .18$ when fitted with a Gaussian function.

The hard X-ray morphology of the NE rim observed by ASCA suggests that the TeV gamma rays may emanate from an extended area over a few times the PSF of the CANGAROO telescope, (several tenths of a degree in extent). Then we approximate the diffuse emission by a superposition of a small number of point sources separated by about $0^{\circ} .3$. In order to search the emission region of $\mathrm{TeV}$ gamma rays in SN1006, significances of peaked events of $\alpha \leq 15^{\circ}$ were calculated at all grid-points in $0^{\circ} .09$ steps in the FOV which is a half pitch of a standard deviation of the PSF. The contour map of significances in 1996 and 1997 data are shown in Figs. 1a and 1b, in which the contours of the hard X-ray flux of ASCA also are overlaid as solid bold-lines. The region showing significant $\mathrm{TeV}$ gamma-ray emission clearly extends along the ridge of the NE rim over the PSF of the telescope, and matches the X-ray image fairly well. The source point in the NE rim giving the most significant $\alpha$ peak $\left(\alpha \leq 15^{\circ}\right)$ was found close to the maximum flux point in the $2-10 \mathrm{keV}$ band of the ASCA data. The statistical significances of these peaks are estimated to be $5.3 \sigma$ in 1996 data and $7.7 \sigma$ in 1997 data at the maximum hard X-ray flux point of ASCA. The much improvement of the detection significance in 1997 data was due to the twice increase of the reflectivity of the mirror. Thus the $\mathrm{TeV}$ gamma-ray emission from the NE rim of SN1006 has been confirmed (Tanimori et al. 1997).

The threshold energies for the 1996 data and 1997 data were determined from Monte Carlo simulations to be $3 \pm 0.9 \mathrm{TeV}$ and $1.7 \pm 0.5 \mathrm{TeV}$, respectively. Although Figs. 1a and $1 \mathrm{~b}$ suggest the emission of $\mathrm{TeV}$ gamma rays extends along the ridge of the $\mathrm{NE}$ rim, we do not yet have a reliable method to estimate the number of gamma-ray events from an extended source. Approximating the emission as coming from a single point source 


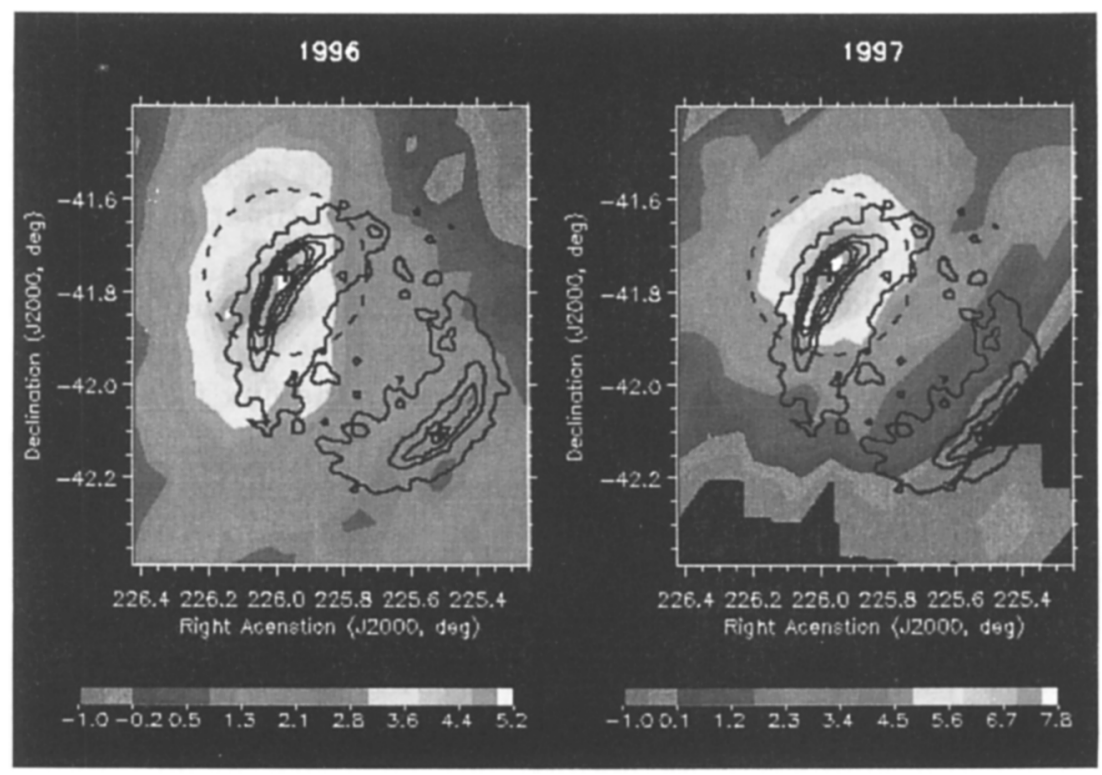

Figure 1. (a) The contour map of statistical significance in the sky around SN1006, where the intensity contours of hard X-rays by ASCA (courtesy M. Ozaki) is overlaid as solid line, and the dashed circle is the PSF of the CANGAROO telescope. (b) The same contour map obtained from the 1997 data.

at the maximum flux point in the NE rim, the integral gamma-ray fluxes for the 1996 and 1997 observations were calculated to be $(2.4 \pm 0.44) \times 10^{-12}$ $\mathrm{cm}^{-2} \mathrm{~s}^{-1}$ and $(4.6 \pm 0.6) \times 10^{-12} \mathrm{~cm}^{-2} \mathrm{~s}^{-1}$, respectively. A larger flux would be obtained for an extended emission wider than the PSF of our detection.

No significant excess is evident in Figs. $1 \mathrm{a}$ and $1 \mathrm{~b}$ near the position of the maximum X-ray flux from the SW rim. We set a upper limit on the $\mathrm{TeV}$ gamma-ray emission from the $\mathrm{SW}$ rim, estimated to be $1.5 \times 10^{-12}$ $\mathrm{cm}^{-2} \mathrm{~s}^{-1}(\geq 3 \pm 0.9 \mathrm{TeV}, 95 \% \mathrm{CL})$ from the $\alpha$ distribution in 1996 data at the position of the maximum ASCA flux in the SW rim.

\section{Discussion}

The detection of $\mathrm{TeV}$ gamma rays from $\mathrm{SN} 1006$ presents a convincing demonstration of the the shock acceleration mechanism for very high energy particles up to $\sim 100 \mathrm{TeV}$ in a SNR. The TeV gamma ray emission region is observed to extend over $\sim 30$ arcmin along the ridge of the NE rim, providing the first detection of very high energy gamma rays from an extended source. From non-thermal X-ray observation, the detected $\mathrm{TeV}$ 
gamma rays are readily presumed to be generated by IC scattering of very high energy electrons and $2.7 \mathrm{~K}$ cosmic background photons. All of the calculated fluxes of $\mathrm{TeV}$ gamma rays based on those assumption are consistent with the obtained $\mathrm{TeV}$ gamma-ray fluxes by assuming the magnetic field strength $(B)$ in the emission region of the SNR with around $6 \mu \mathrm{G}$. This strength of $B$ is well consistent with the previous estimation of $6-10 \mu \mathrm{G}$ (Reynolds 1991).

The other candidate of the production mechanism of the TeV gammaray emission is a decay of neutral pions induced by high energy protons accelerated in the SNR. However, we can neglect the flux from the $\pi^{0}$ decay due to following arguments. Since SN1006 (G327.6+14.6) is located above the galactic plane, the matter density at the shock is low $(\sim 0.05$ $\mathrm{cm}^{-3}$ ) so that the expected flux will be about a factor of ten less than the observed flux (Mastichiadis 1996). The upper limit for GeV gamma-ray emission from the EGRET archive data is also consistent with the IC model. Thus, the detected gamma-rays are likely to be explained by IC radiation from electrons, and our result testifies to the existence of the very high energy electrons of more than several times $10 \mathrm{TeV}$ in SN1006. The highest energy of non-thermal electrons can be estimated from bending point in synchrotron spectrum and the resultant magnetic fields. Although the bending energy of SN1006 is not precisely determined yet in recent observations, $1 \mathrm{keV}$ photon from synchrotron radiation corresponds to electron energy of $\sim 60 \mathrm{TeV}$ for $B \sim 6 \mu \mathrm{G}$. On the hand, there are no distinctions between electrons and protons for the basic theoretical treatment of shock acceleration via first-order Fermi process. Our observation is therefore the first convincing proof of the shock acceleration of high energy cosmic rays in a SNR.

\section{References}

Hara, T. et al. (1993), Nuc. Inst. Meth. Phys. Res. A, 332, $300-309$

Kifune, T. et al. (1995), Astrophys. J., 438, L91 - L94

Koyama, K. et al. (1995), Nature, 378, $255-258$

Mastichiadis, A. (1996); Astr. Astrophys., 305, L53 - L56

Mastichiadis, A. \& de Jager, O.C. (1996), Astr. Astrophys., 311, L5 - L8

Patterson, J. R., \& Kifune, T. (1992), Australian and New Zealand Physicist, 29, 58 - 62

Pohl, M. (1996), Astr. Astrophys., 307, 57 - 395

Reynolds, S.P. \& Ellison, D.C. (1991), Proc. 22nd Int. Cosmic ray Conf., 2 404-407

Tanimori, T. et al. (1997), IAU Circ. No. 6706

Tanimori, T. et al. (1998), Astrophys. J., in press

Yoshida, T.\& Yanagita, S. (1997), Proc. 2nd INTEGRAL Workshop, ESA SP382 85-88

Weekes, T.C. et al. (1989), Astrophys. J., 342, $379-395$

Willingale, R. et al. (1996) Mon. Not. R. astr. Soc., 278, $749-762$ 